IMAGING

\title{
Can hysterosalpingo-contrast sonography replace hysterosalpingography in confirming tubal blockage after hysteroscopic sterilization and in the evaluation of the uterus and tubes in infertile patients?
}

\author{
Danielle E. Luciano, MD; Caterina Exacoustos, MD; D. Alan Johns, MD; Anthony A. Luciano, MD
}

OBJECTIVE: The objective of the study was to assess the accuracy of hysterosalpingo-contrast sonography (HyCoSy) in establishing tubal patency or blockage and evaluating the uterine cavity by comparing it with hysteroscopy laparoscopy (HLC) or hysterosalpingography (HSG).

STUDY DESIGN: This study was a chart review evaluating infertility patients and patients who had undergone hysteroscopic sterilization who underwent both HyCoSy and HLC or HyCoSy and HSG at private offices associated with university hospitals. Sensitivity, specificity, positive predictive value, and negative predictive value of HyCoSy were calculated.
RESULTS: HyCoSy compared with HLC had a sensitivity of $97 \%$ and specificity of $82 \%$, and HyCoSy compared with HSG was $100 \%$ concordant. Uterine cavities evaluated by sonohysterography and hysteroscopy were $100 \%$ concordant.

CONCLUSION: HyCoSy is accurate in determining tubal patency and evaluating the uterine cavity, suggesting it could supplant HSG not only as the first-line diagnostic test in an infertility workup but also in confirming tubal blockage after hysteroscopic sterilization.

Key words: chromopertubation, hysterosalpingo-contrast sonography, hysterosalpingogram, infertility, tubal sterilization

Cite this article as: Luciano DE, Exacoustos C, Johns DA, et al. Can hysterosalpingo-contrast sonography replace hysterosalpingography in confirming tubal blockage after hysteroscopic sterilization and in the evaluation of the uterus and tubes in infertile patients? Am J 0bstet Gynecol 2011;204:79.e1-5.

$\mathrm{T}$ he evaluation of the fallopian tubes and the endometrial cavity is an essential part of the infertility workup. Currently this is done either by hysterosalpingography (HSG) or at the time of hysteroscopy and laparoscopy with chromopertubation (HLC). HLC is considered the gold standard for assessing the integrity of the uterine cavity and for establishing tubal status. It allows the physician to diag-

From the Center for Fertility and Women's Health, New Britain, CT (Drs D. E. Luciano and A. A. Luciano); the Department of Obstetrics and Gynecology, University of Rome "Tor Vergata," Rome, Italy (Dr Exacoustos); and the University of Texas Southwestern Medical Center, Fort Worth, TX (Dr Johns).

Received June 4, 2010; revised July 27, 2010; accepted Aug. 20, 2010.

Reprints: Danielle E. Luciano, MD, Center for Fertility and Women's Health, 100 Grand St., Suite E3, New Britain, CT 06050. dluciano@aol.com.

0002-9378/\$36.00

(C) 2011 Mosby, Inc. All rights reserved. doi: 10.1016/j.ajog.2010.08.065 nose and treat the problem. When compared with HLC, HSG has been reported to have a sensitivity of $72-85 \%$ and a specificity of $68-89 \%$ in diagnosing tubal patency. ${ }^{1,2,3}$ HSG is also currently required to confirm tubal blockage 3 months after hysteroscopic tubal sterilization.

Hysterosalpingo-contrast sonography (HyCoSy) uses positive ultrasound-enhancing contrast media with transvaginal ultrasound to assess the status of the fallopian tubes, as well as the uterine cavity, by means of conventional sonohysterography. It can be performed in the gynecologist's office, in which it is more convenient for the patient and the gynecologist. Initial studies used air and saline as the contrast agent, with or without Doppler, but further studies began using ultrasound-dedicated contrast media. The air bubbles and microbubbles of these first-generation agents diffuse very quickly, leading to a short duration of visualization of the contrast fluid, making the test more difficult and requiring greater operator experience to obtain consistently reliable results.

Second-generation agents, such as Sonovue (BR-1, sulfur hexafluoride; Bracco
International BV, Amsterdam, The Netherlands) and Definity (MRX-115, perflutren lipid microspheres; Bristol MyersSquibb Medical Imaging, North Billerica, MA), use gases with less diffusibility and solubility and have a longer duration of visualization, allowing for easier evaluation of the tubal course. ${ }^{4}$ However, bowel gas and hyperechoic signals coming from pelvic organs may interfere with the signals from these newer contrast media.

To minimize interference from other pelvic organs, a special ultrasound software (contrast-tuned imaging [CnTI] Biosound ESAOTE, Indianapolis, IN), which picks up only the sound waves from the contrast medium and ignores surrounding structures, has been developed. With the CnTI technology, the course of the contrast fluid is followed as a white band in a black background flowing from the endometrial cavity into the fallopian tubes and the dark peritoneal cavity. Although CnTI technology is not required to perform HyCoSy, it has been shown to have better accuracy in determining tubal patency. However, in a previous study using Sonovue, only small numbers of tubes 


\section{FIGURE 1}

\section{HyCoSy demonstrating tubal patency and tubal blockage}
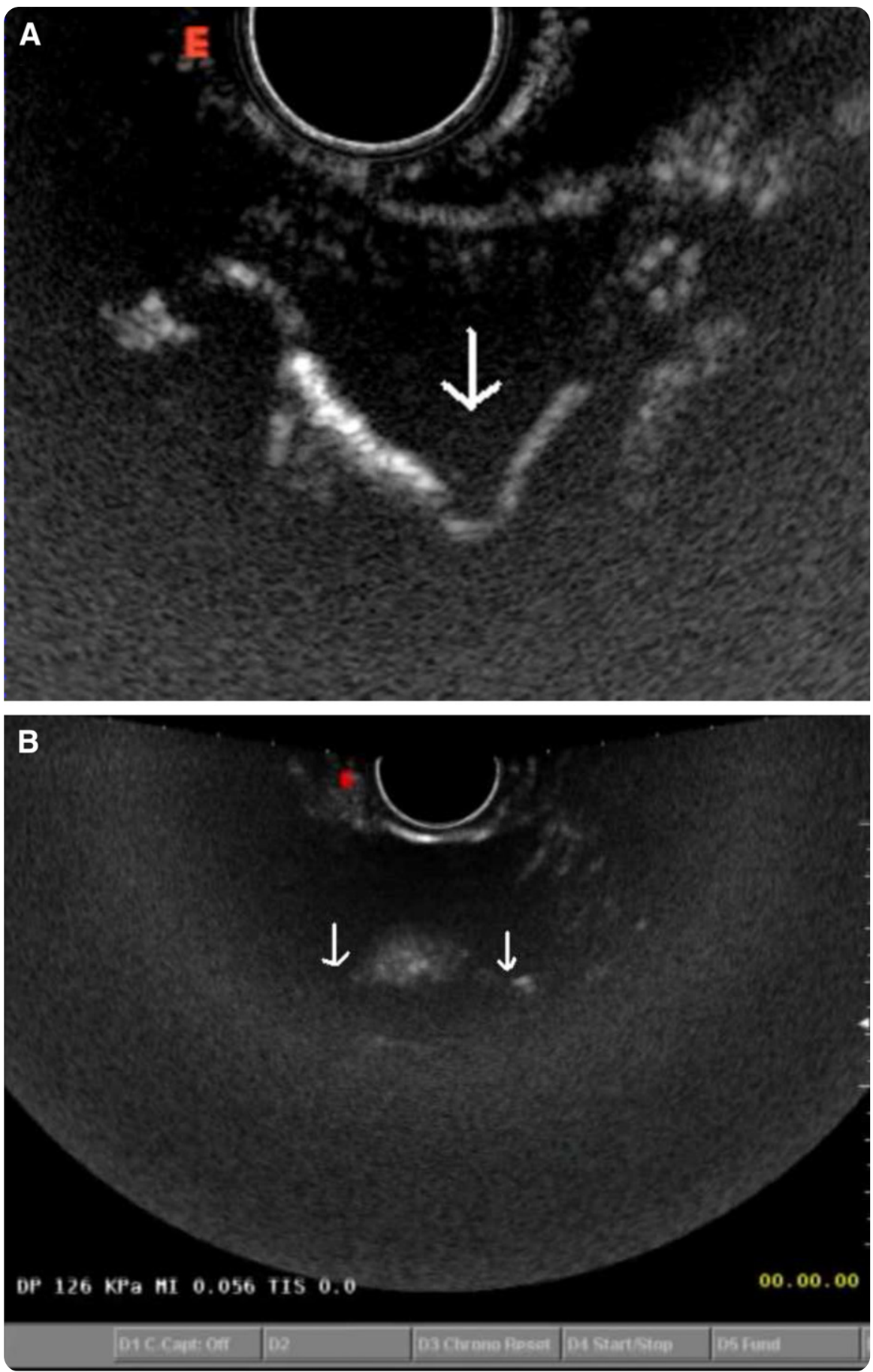

A, Right tubal patency. B, Bilateral tubal blockage following hysteroscopic sterilization.

HyCoSy, hysterosalpingo-contrast sonography.

Luciano. Hysterosalpingo-contrast sonography to evaluate tubal patency. Am J Obstet Gynecol 2011.

were evaluated by both HyCoSy and HLC, and less accuracy on tubal occlusion was reported. ${ }^{5}$ Although Sonovue is not approved by the Food and Drug Administration (FDA) for use in the United States, a similar contrast media, Definity, is FDA approved and currently indicated for intravenous infusion during echocardiography, although it is not currently indicated for HyCoSy.
The aim of this study was to assess the accuracy of HyCoSy using CnTI technology and Definity contrast media in the evaluation of the fallopian tubes and the endometrial cavity by comparing it with HLC or HSG.

\section{Materials and Methods}

Following the introduction of HyCoSy and CnTI technology in the 2 US centers (Center for Fertility and Women's Health, New Britain, CT, and the University of Texas Southwestern Medical Center, Fort Worth, TX), data were collected on all patients (200) who underwent HyCoSy from July 2007 through December 2009. Both US centers are accredited by the American Institute of Ultrasound in Medicine.

Patients were included in this study if they had a HyCoSy as part of an infertility evaluation and then had either an HSG or an HLC (if surgery was indicated for the treatment of endometriosis, pelvic adhesions, uterine malformations, or fibroids).

Patients who were scheduled for HSG, to confirm tubal blockage after hysteroscopic tubal sterilization, were consented to undergo a HyCoSy, as well as an HSG. Institutional review board approval was obtained at both institutions at which these procedures and this study were conducted. The patients were not compensated for their participation, and the costs of the HyCoSy were offset by the clinic.

HyCoSy was performed as previously described. ${ }^{1}$ Definity contrast media was activated by agitation in a dental shaker for 45 seconds. One milliliter of Definity media was mixed with $9 \mathrm{~mL}$ of injectable sterile normal saline in a $10 \mathrm{~mL}$ syringe. After cleaning the patients' cervix with betadine, a 5 French HSG catheter (Rocket Medical, Hingham, MA) was placed inside the cervix into the lower uterine cavity and the catheter balloon was inflated.

Transvaginal ultrasound was performed using an EC1239-3 vaginal probe on the Biosound ESAOTE Technos MPX ultrasound machine to visualize the uterus, the ovaries, and the proper placement of the HSG catheter. CnTI software was activated while Definity solution was being injected through the catheter into the uterine cavity. The hyperechoic bub- 


\section{FIGURE 2 \\ Laparoscopy chromopertubation, establishing patency of right tube}

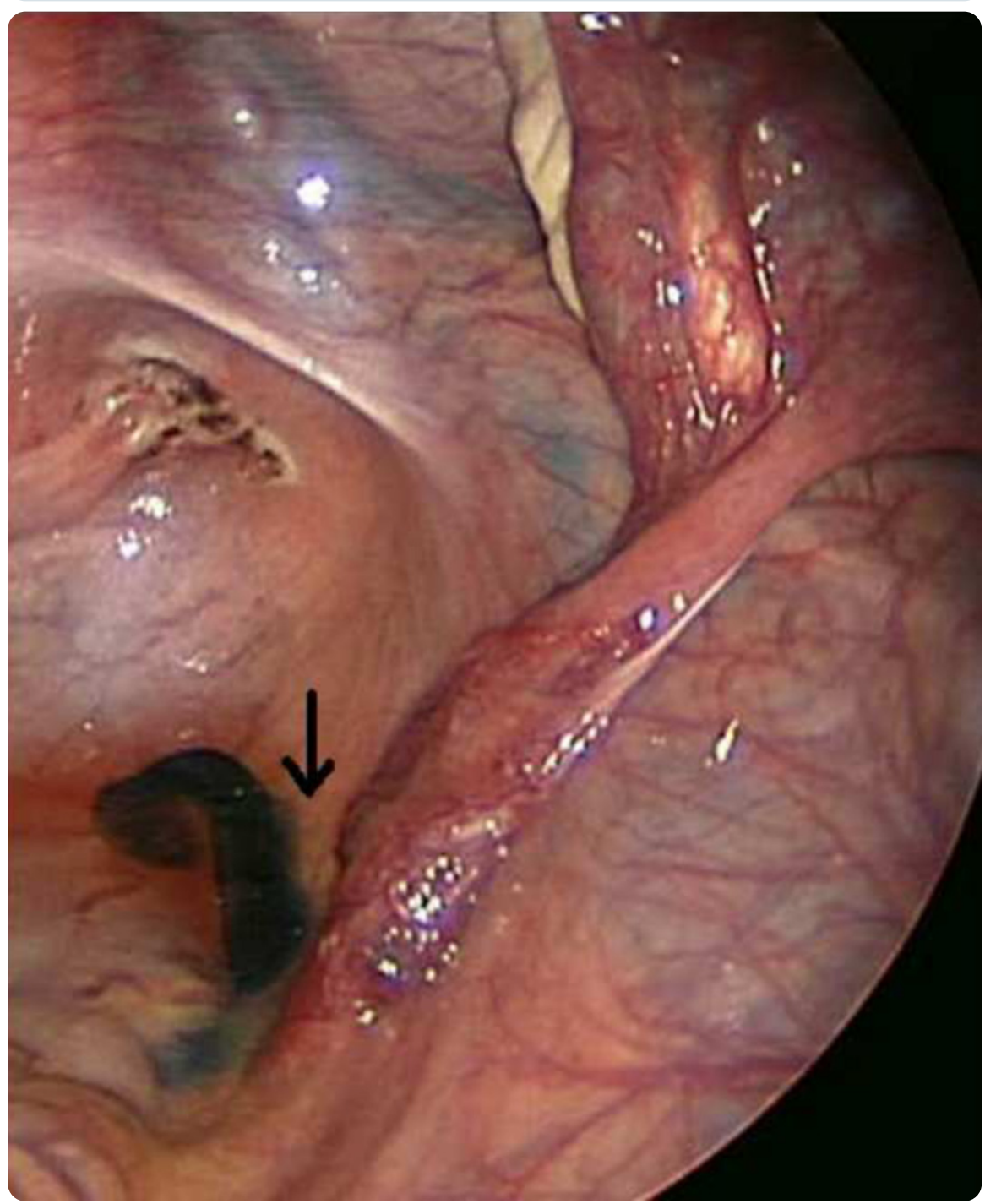

Luciano. Hysterosalpingo-contrast sonography to evaluate tubal patency. Am J Obstet Gynecol 2011.

bles were visualized on the screen as they filled the endometrial cavity and followed the course of the tubes from each cornua, around the ipsilateral ovary, and into the peritoneal cavity when the tubes were patent. When the tubes were blocked, the contrast advanced to the point of blockage, as shown in Figure 1, $\mathrm{A}$ and $\mathrm{B}$. When the tubal evaluation was completed, the media were switched to normal saline, the balloon of the catheter was deflated, and $10-30 \mathrm{~mL}$ of saline was infused to evaluate the uterine cavity in the usual fashion of sonohysterography.

To minimize pelvic discomfort from uterine cramps, the patients were ad- vised to take $400 \mathrm{mg}$ of ibuprofen 1-2 hours prior to the procedure.

During the hysteroscopy and laparoscopy (HLC), the patients had a full evaluation of the uterine cavity, and chromopertubation was performed using indigo carmine solution injected through a Clear View uterine manipulator (Clinical Innovations, Murray, UT). Endometriosis, adhesions, fibroids, and adnexal masses were then treated and removed as indicated. Results of HLC were compared with HyCoSy, and representative photos confirming tubal patency or blockage were taken (Figure 2).
The HSG was performed by instilling Sinografin (diatrizoate meglumine and lodipamide meglumine injection; Bracco Diagnostics Inc, Princeton, NJ) into the endometrial cavity using a 5 French HSG catheter (Rocket Medical) under fluoroscopic visualization. Representative photographs were obtained, and the HSG films were reviewed by the radiologist and then compared with those obtained with HyCoSy.

Statistical analyses were conducted to assess whether the tubes found to be patent or occluded by HyCoSy were also found to have the same status at laparoscopic chromopertubation or at HSG. Baye's theorem was used to evaluate the diagnostic accuracy for tubal patency of HyCoSy. Sensitivity, specificity, accuracy, and negative and positive predictive values were calculated. Comparison between hysteroscopic and sonohysterographic findings was performed and concordance rate calculated.

\section{RESUlTS}

In 62 patients, 121 tubes and 58 uterine cavities were evaluated by both HyCoSy and HLC. One patient had a unicornuate uterus with only 1 tube, and 2 patients were status postunilateral salpingectomy. Four patients were undergoing tubal reanastomosis and did not have a hysteroscopy as part of the procedure. At laparoscopy the primary diagnosis for 17 patients was endometriosis, 16 patients had adhesive disease, and 3 patients had fibroids.

Thirty-six patients (72 tubes) were evaluated by HyCoSy and HSG for infertility evaluation, and 15 patients (30 tubes) were evaluated by HyCoSy and HSG for tubal blockage following hysteroscopic sterilization. The distribution of patients undergoing HyCoSy, laparoscopy chromopertubation (LC), hysteroscopy, and HSG is depicted in the flow chart of Figure 3.

Of the 121 tubes evaluated by both HyCoSy and HLC, 110 tubes were concordant and 11 were not concordant (Table). At laparoscopic chromoperturbation 71 tubes were patent and $50 \mathrm{oc}-$ cluded. The sensitivity and specificity for HyCoSy in determining tubal patency compared with HLC were $97 \%$ and $82 \%$, 


\section{FIGURE 3}

\section{Flowchart of the diagnostic procedures}

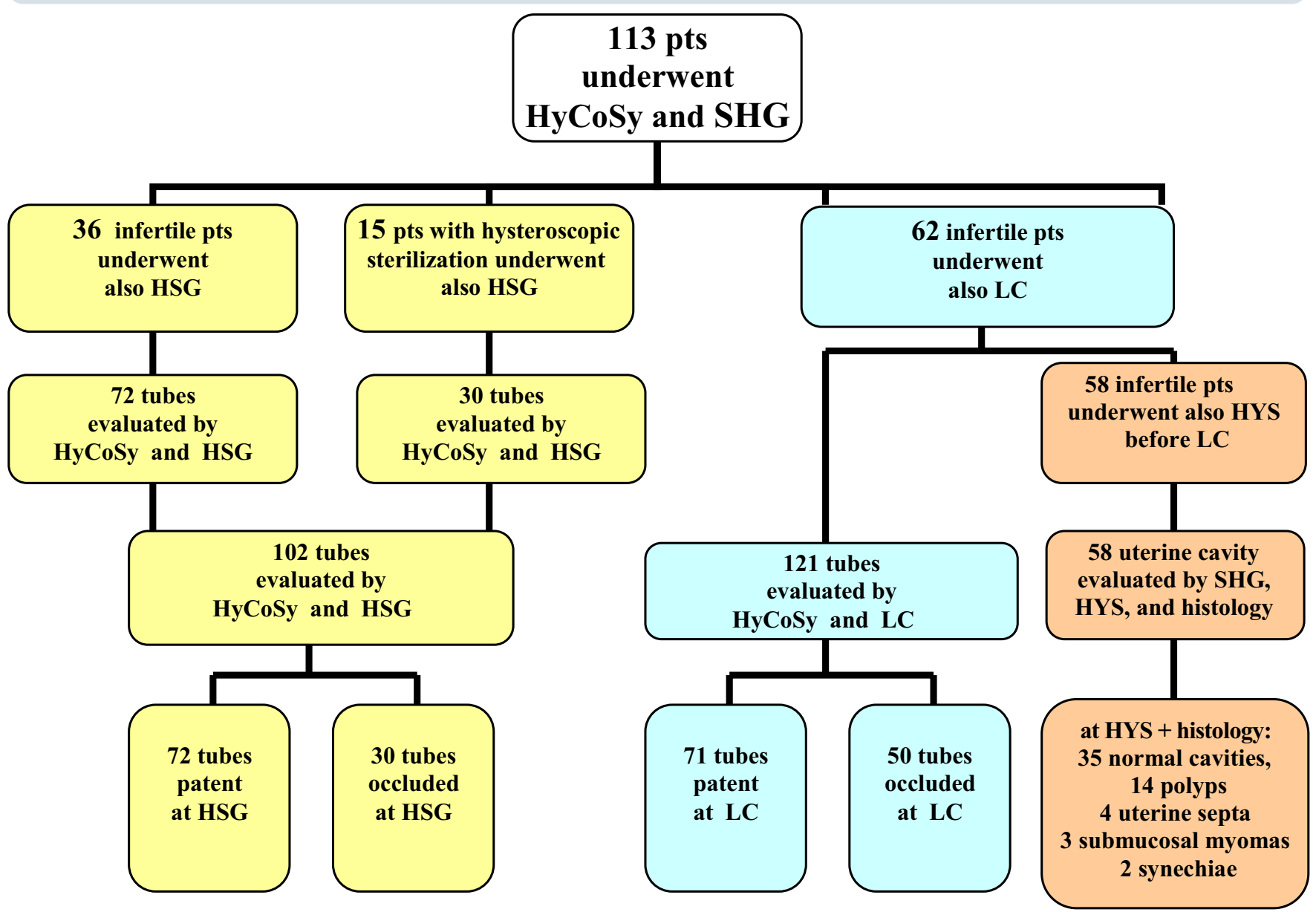

Flowchart of the diagnostic procedures performed in our study population to evaluate the tubal patency and uterine cavity. Yellow color indicates patients evaluated by HyCoSy and HYS. Blue colorindicates patients evaluated by HyCoSy and LC. Pink colorindicates patients evaluated by SHG after HyCoSy and HYS before LC.

HyCoSy, hysterosalpingo-contrast sonography; HSG, hysterosalpingography; HYS, hysteroscopy; LC, laparoscopy with chromopertubation; SHG, sonohysterography.

Luciano. Hysterosalpingo-contrast sonography to evaluate tubal patency. Am J Obstet Gynecol 2011.

respectively; the positive predictive value (PPV) was $88 \%$ and the negative predictive value (NPV) was 95\%. Nine tubes were seen as patent by HyCoSy but showed occlusion at HLC because of adhesions in 2 cases and adherent fimbriae in 2 cases. In the other 2 patients, chromopertubation was unable to be performed because of malfunctioning of the uterine manipulator.

Of the 102 tubes evaluated by both HyCoSy and HSG, 22 were patent and 30 were occluded. For HSG and HyCoSy, all 102 tubes were concordant. Of the 15 patients who underwent tubal sterilization, all showed occluded tubes 3 months after the procedure.
Of the 58 uterine cavities evaluated by sonohysterography and hysteroscopy, all cavities were concordant. At hysteroscopy the results on sonohysterography were confirmed; 35 patients had normal cavities, 14 had endometrial polyps, 2 had uterine synechiae, 3 had submucosal fibroids, and 4 had uterine septa.

Two patients had minor vasovagal reactions (dizziness and light headedness) following the HyCoSy that required minimal supportive measures, including oral intake of fluid and resting for 5-10 minutes in the supine position; their symptoms resolved. No allergic reactions to the contrast media were ob- served. No untoward events occurred during any of the surgeries.

\section{Comment}

Our study shows that HyCoSy performed with Definity and CnTI has a similar sensitivity and specificity for tubal patency as HSG, without the inconvenience and potential hazards of HSG, such as possible iodine-based sensitivity to the radiopaque dye and exposure to radiation. HyCoSy can be performed in the gynecologist's office, and by using the ultrasound, a full evaluation of the uterus, ovaries, and pelvis can be performed at the same time. Intrauterine 


\section{TABLE}

\section{Accuracy of HyCoSy compared with LC and HSG}

\begin{tabular}{|c|c|c|}
\hline \multirow[b]{2}{*}{ Variable } & \multicolumn{2}{|c|}{ Accuracy for tubal patency } \\
\hline & HyCoSy vs LC & HyCoSy vs HSG \\
\hline & 62 patients & 51 patients \\
\hline & 121 tubes & 102 tubes \\
\hline True positive & 69 & 72 \\
\hline False positive & 9 & 0 \\
\hline True negative & 41 & 30 \\
\hline False negative & 2 & 0 \\
\hline Prevalence patent tubes & $59 \%$ & $71 \%$ \\
\hline Sensitivity & $97 \%$ & $100 \%$ \\
\hline Specificity & $82 \%$ & $100 \%$ \\
\hline PPV & $88 \%$ & $100 \%$ \\
\hline NPV & $95 \%$ & $100 \%$ \\
\hline Accuracy & $91 \%$ & $100 \%$ \\
\hline
\end{tabular}

defects can be identified and accurately diagnosed as polyps, fibroids, or adhesions rather than vague filling defects. By supplanting HSG with HyCoSy, we would be in compliance with the recent initiative of the FDA to reduce unnecessary radiation exposure from computed tomography, nuclear medicine studies, and fluoroscopy.

The advantages of HyCoSy over HSG in the evaluation of the infertile patients are equally valid in the evaluation of patients following hysteroscopic sterilization. Currently in the United States, 2 devices are FDA approved for hysteroscopic sterilization, Essure (Conceptus Inc) and Adiana Permanent Contraception System (Hologic Inc). They both require confirmation of tubal blockage by HSG 3 months after sterilization.

Our data show excellent concordance of HyCoSy with HSG in confirming tubal blockage, but HyCoSy is more convenient for the patients and often less uncomfortable. In a similar study to ours, Connor reported excellent concordance (31/33 tubes) between HyCoSy and HSG; in addition, her patients experienced less discomfort with the HyCoSy and expressed that "the confirmatory test performed by their gynecologist in the familiar office setting was preferable to the one performed by an unfamiliar physician in radiology." 6

Of the 700,000 tubal sterilizations performed in the United States annually if, as predicted, most of them will switch to hysteroscopic sterilization, the use of HyCoSy instead of HSG will significantly reduce unnecessary radiation exposure to women, as well as reduce costs.

Another innovation that was studied and applied in this study was CnTI technology, which allows for better visualization of the tubes by eliminating the echoes from other pelvic organs and structures. Although CnTI software is not essential for performing HyCoSy, it makes HyCoSy an easier diagnostic test that can be successfully performed by less experienced sonographers. When compared with HLC, which is considered the gold standard test for tubal patency, our study demonstrated that HyCoSy has a high sensitivity in evaluating tubal patency; however, specificity and PPV were lower because of the 9 cases with false-positive results.

The fact that contrast fluid passage in the tube was observed during HyCoSy and not during HLC can be explained by distal tubal blockage and by spillage in the cavity around the ovaries from the contralateral tube. A tubal spasm during chromoperturbation also because of previous hysteroscopic procedures, however, can not be totally excluded. In comparison with previous studies, we are more accurate in evaluating tubal occlusions, with an NPV of $95 \%$ and $100 \%$ for both study groups.

The high concordance of sonohysterography and hysteroscopy obtained in this study confirmed the value of this test in the evaluation of uterine cavity, reducing time, costs, and discomfort for the patients.

In conclusion, HyCoSy with CnTI technology and Definity as the contrast media, is as accurate and as reliable as HSG in the evaluation of the endometrial cavity and in establishing tubal patency or blockage. When compared with HLC, HyCoSy is similarly reliable in the evaluation of the uterine cavity and fallopian tubes. The high accuracy of HyCoSy shown in this study suggests that it may supplant HSG not only as the first-line diagnostic test in the infertility workup but also in confirming tubal blockage after hysteroscopic sterilization.

\section{REFERENCES}

1. Exacoustos C, Zupi E, Carusotti C, Lanzi G, Marconi D, Arduini D. Hysterosalpingo-contrast sonography compared with hysterosalpingography and laparoscopic dye perturbation to evaluate tubal patency. J Am Assoc Gynecol Laparosc 2003;10:367-72.

2. Adelusi B, Al-Nuaim L, Makanjuoal D, Chowdhury N, Kangave D. Accuracy of hysterosalpingography and laparoscopic hydrotubation in diagnosis of tubal patency. Fertil Steril 1995;63:1016-20.

3. Dijkman AB, Mol BWJ, van der Veen F, Bossuyt PMM, Hogerzeil HV. Can hysterosalpingocontrast-sonography replace hysterosalpingography in the assessment of tubal subfertility? Eur J Radiol 2000;35:44-8.

4. Miller DL, Averkiou MA, Brayman AA, et al. Bioeffects considerations for diagnostic ultrasound contrast agents. J Ultrasound Med 2008;27:611-32.

5. Exacoustos C, Zupi E, Szabolcs B, et al. Contrast-tuned imaging and second generation contrast agent SonoVue: a new ultrasound approach to evaluation of tubal patency. J Min Inv Gynecol 2009;16:437-44.

6. Connor V. Contrast infusion sonography in the post Essure setting. J Min Inv Gynecol 2008;15:56-61. 